\title{
Contribuição ao estudo da biologia de Biomphalaria occidentalis Paraense, 1981 e de Biomphalaria tenagophila (d'Orbigny, 1835), em condições de laboratório
}

\section{A contribution to the study of the biology of Biomphalaria occidentalis Paraense, 1981 and of Biomphalaria tenagophila (d'Orbigny, 1835), under laboratory conditions}

\author{
Maisa Rose Domenico Elmor*, Jorge Faria Vaz*, Léa Maria Cappelletti Gonçalves* \\ ELMOR, M.R.D. et al. Contribuição ao estudo da biologia de Biomphalaria occidentalis \\ Paraense, 1981 e de Biomphalaria tenagophila (d'Orbigny, 1835), em condições de laboratório, \\ Rev. Saúde públ., S.Paulo, 26: 343-9, 1992. Com o objetivo de comparar a duração do período \\ embrionário, a fecundidade e a fertilidade de Biomphalaria occidentalis Paraense, $1981 \mathrm{com}$ \\ a de $B$. tenagophila (d'Orbigny, 1835), exemplares de ambas as espécies foram criados em \\ aquários dotados das mesmas características. Os ovos depostos pelos caramujos foram contados \\ sob lupa binocular e seu desenvolvimento embrionário foi observado até a eclosão. Assim foi \\ obtido o número total de posturas e de ovos por caramujo, bem como o número total de ovos \\ eclodidos por postura para cada período de trinta dias, ou seja, a taxa de eclosão por período. \\ O experimento teve a duração de doze meses e os resultados obtidos são válidos para condi- \\ çőes de laboratório.
}

Descritores : Biomphalaria, fisiologia. Oviposiçåo.

\section{Introdução}

O conhecimento da biologia dos representantes do gênero Biomphalaria é de grande importância para todos os que se interessam pelo controle da esquistossomose, endemia que acomete grande parte da população de nosso país.

Vários autores volveram sua atenção para o estudo da biologia dos hospedeiros intermediários do Schistosoma mansoni, havendo cada um deles contribuido para esclarecer facetas do problema, mas continuam surgindo outras, aguardando elucidação. Uma delas é a possibilidade de Biomphalaria occidentalis Paraense ${ }^{7}$ (1981) vir a ser empregada no controle das espécies vetoras, porquanto nāo só é refratária à infecçāo pela cepa SJ-2 de $S$. mansoni, conforme mostraram Paraense e Correa ${ }^{8}$ (1982), mas também porque a evidência epidemiológica obtida por Vaz e col. ${ }^{14}$ (1983), em São Paulo, leva a crer que a mesma não se infecte com todas as demais cepas do trematódeo.

A busca de informações sobre a biologia da nova espécie motivou o presente trabalho,

* Laboratório de Malacologia da Superintendência de Controle de Endemias (SUCEN) - São Paulo, SP - Brasil

Separatas/Reprints: J.F.Vaz - Alameda Lorena, 1919 - Jardim Paulista - 01424-002 - São Paulo, SP - Brasil

Publicação financiada pela FAPESP. Processo Saúde Coletiva 91/4994-0 realizado no laboratório de Malacologia da Superintendência de Controle de Endemias (SUCEN). Comparam-se os dados referentes a $B$. occidentalis e a $B$. tenagophila no que tange à fertilidade, à fecundidade e ao tempo de desenvolvimento embrionário.

Os resultados obtidos representam uma abordagem inicial do problema, ainda totalmente aberto a novas e mais precisas' investigações.

\section{Material e Método}

Foram utilizados exemplares de $B$. occidentalis de Presidente Prudente, SP, e representantes de $B$. tenagophila de Ribeirão Pires, SP. Os primeiros, após a coleta foram secos em papel toalha, envoltos em gaze não umedecida, embalados em caixas de papelão e remetidos para o Município de São Paulo, por rodovia. Os caramujos das imediações desse Município, depois de colhidos, foram postos em copos de plástico e, em seguida, transportados para o Laboratório de Malacologia em viaturas do próprio serviço.

Para estudar a fecundidade e a fertilidade de $B$. occidentalis foram selecionados dezesseis exemplares com o diâmetro de 13,8 a 3,8 $\mathrm{mm}$, que foram distribuídos em parcelas iguais por quatro aquários. 
Para comparar a fecundidade e fertilidade de $B$. occidentalis com a de $B$. tenagophila, foram utilizados dois pares de cada espécie, com diâmetros médios das conchas iguais a $13,8 \pm 0,8 \mathrm{~mm}$ e $11,1 \pm 1,4 \mathrm{~mm}$, respectivamente, colocado cada par em um aquário. Estes, com a capacidade de $1.300 \mathrm{ml}$ cada, eram cilíndricos, de vidro, desprovidos de substrato arenoso e de plantas aquáticas, o que muito facilitou a observação das posturas.

A água utilizada era potável, não clorada, e foi trocada semanalmente.

$\mathrm{O}$ alimento fornecido todos os dias em retângulos de isopor constou da ração balanceada para aves do Moinho Fluminense Avevita $X$ preconizada por Oliveira e col. ${ }^{6}$ (1968), enriquecida com alfatocoferol na proporção de $0,6 \mathrm{mg}$ por $50 \mathrm{gr}$, conforme proposta de Barnett e col. ${ }^{1}$ (1974).

Os caramujos deitaram suas posturas de preferência na face inferior dos retângulos de isopor flutuantes que eram usados como suporte de alimento. Depuseram também sobre as paredes internas do recipiente e sobre as conchas de seus parceiros.

Todas as manhãs, as cápsulas ovíferas eram destacadas do isopor e, com o auxílio de uma lâmina afiada, eram retiradas dos outros locais, e colocadas em um pequeno béquer de $80 \mathrm{ml}$, com água pela metade. Os ovos assim coletados eram contados sob lupa binocular e o desenvolvimento embrionário era observado até a eclosão. Dessa maneira, foi possível conhecer o número total de posturas e o número total de ovos por caramujo, bem como o número de ovos eclodidos por postura em cada periodo de trinta dias. Os dados obtidos permitiram calcular a taxa de eclosão e o número de ovos depostos em cada período de trinta dias.
O experimento durou doze meses e o prazo máximo para a observação das posturas foi de trinta dias, após os quais os ovos que não chegaram à eclosão eram considerados estéreis.

Três vezes ao dia foram medidas as temperaturas do ar, da água dos aquários e da água de um béquer semelhante ao utilizado para a conservação das posturas, o que permitiu obter a média diária das temperaturas.

Cada exemplar morto no decorrer do experimento foi substituído por outro do mesmo tamanho e procedência. Convém notar que em trabalhos da mesma natureza não há referência a essa conduta, salvo em Chieffi e col. ${ }^{2}$ (1977) e em Genaro $^{3}$ (1978).

\section{Resultados}

\section{Duraçāo do período embrionário}

Os resultados obtidos para cada espécie figuram na Tabela 1, na qual deixaram de ser consignados os dois primeiros meses por ter havido falhas no registro que só foram corrigidas a partir de dezembro.

$O$ desenvolvimento embrionário em $B$. tenagophila variou de 6,5 a 18,7 dias e o de $B$. occidentalis, entre 7,1 e 16,6 dias.

As mais altas temperaturas do meio ambiente foram observadas em dezembro, janeiro, fevereiro e março e coincidiram, em ambas as espécies, com os menores períodos de desenvolvimento embrionário.

Em B. occidentalis, o embriāo desenvolveu-se mais lentamente em julho e setembro, meses de baixas temperaturas.

No mês de maio, coisa semelhante ocorreu com B. tenagophila, conforme mostra a Tabela 1.

Tabela 1. Duração do período embrionário de B. tenagophila e de B. occidentalis. Anos de 1981 e 1982. Posturas de 16 exemplares de cada espécie

\begin{tabular}{|c|c|c|c|c|c|c|}
\hline \multirow[t]{2}{*}{ Mês } & \multicolumn{2}{|c|}{$\begin{array}{l}\text { Duração do periodo } \\
\text { embrionário ( } X \text { dias) }\end{array}$} & \multicolumn{2}{|c|}{$\begin{array}{l}\text { Número de postura } \\
\text { observadas }\end{array}$} & \multicolumn{2}{|c|}{$\begin{array}{l}\text { Temperatura média } \\
(\mathrm{C})\end{array}$} \\
\hline & B. tenagophila & B. occidentalis & B. tenagophila & B. occidentalis & "beaker" & ar \\
\hline dezembro & 6,6 & 8,6 & 14 & 89 & 23,2 & 24,2 \\
\hline janeiro & 8,7 & 9,9 & 13 & 82 & 22,7 & 23,4 \\
\hline tevereiro & 6,5 & 7,1 & 14 & 21 & 24,7 & 26,0 \\
\hline março & 7,5 & 8,9 & 13 & 89 & 24,1 & 24,5 \\
\hline abril & 9,7 & 14,1 & 22 & 38 & 22,7 & 22,2 \\
\hline maio & 18,7 & 13,7 & 11 & 15 & 21,5 & 22,4 \\
\hline junho & 12,7 & 10,8 & 05 & 33 & 21,8 & 22,5 \\
\hline julho & 12,2 & 16,6 & 10 & 47 & 21,9 & 22,8 \\
\hline agosto & 10,2 & 13,7 & 12 & 13 & 21,9 & 22,9 \\
\hline setembro & 11,5 & 14,8 & 16 & 25 & 22,1 & 23,1 \\
\hline
\end{tabular}




\section{Fecundidade e Fertilidade}

Conforme já foi dito, só dois autores entre os consultados procediam à reposição dos exemplates mortos ao estudar a biologia dos planorbídeos.

As informações sobre a fecundidade de $B$. occidentalis foram obtidas de duas maneiras: a) deixando à margem as substituiçōes feitas; e b) levando-as em conta, o que permitiu comparar os resultados conseguidos.

$\mathrm{Na}$ tabela 2 foram consignadas as somatórias dos dados sobre os quatro lotes de $B$. occidentalis durante doze períodos de trinta dias. Não foram levadas em consideração as substituiçōes, admitindo-se que dezessejs exemplares sempre estivessem presentes no total de lotes. As cifras mostram que $B$. occidentalis depositou, em média, 8,7 posturas por caramujo, em cada período de trinta dias, o que corresponde a 0,29 posturas por caramujo, por dia. O número de ovos por caramujo, por dia, foi igual a 5,62 .
As oscilações térmicas ambientais parecem nāo ter exercido influência sobre a oviposição, o que é evidenciado na Tabela 3, na qual se observam três picos correspondentes aos meses de dezembro-janeiro, março-abril e agosto-setembro.

A taxa de eclosão, cuja média por periodo foi de $44,43 \%$, oscilou muito tendo sido observados os menores valores nos três últimos períodos, o que é bem perceptível na Tabela 2 .

A Tabela 3 permite comparar os dados de $B$. occidentalis com os de B. tenagophila. Nela foi considerado o número total de indivíduos, levando-se em conta a substituição dos caramujos mortos durante o experimento.

O décimo segundo período não foi relacionado porque ao término da pesquisa desenvolveram-se fungos em algumas cápsulas ovíferas.

Os dados mostram que cada exemplar de $B$. tenagophila deitou, em média, 65 posturas nos onze períodos, ou seja, 5,9 por período. A taxa de eclosão de $B$. tenagophila foi igual a $58,3 \%$, por período.

Tabela 2. Fecundidade e fertilidade, para periodos de 30 dias, de Biomphalaria occidentalis. Posturas de 16 exemplares

\begin{tabular}{|c|c|c|c|c|c|c|c|}
\hline \multirow[b]{2}{*}{$\begin{array}{r}\text { Periodo } \\
\text { (30 dias) }\end{array}$} & \multicolumn{2}{|l|}{ Postura } & $N^{2}$ de ovos & \multirow{2}{*}{$\begin{array}{l}N^{2} \text { total } \\
\text { de ovos } \\
\text { embriona- } \\
\text { dos }\end{array}$} & \multicolumn{2}{|c|}{$N^{2}$ de ovos eclodidos } & \multirow{2}{*}{$\begin{array}{c}\text { Taxa de } \\
\text { eclosão } \\
(\%)\end{array}$} \\
\hline & Ne total & $\begin{array}{c}\text { Postura } \\
\text { p/caramujo }\end{array}$ & Total & & Total & $\begin{array}{c}\text { ovos } \\
\text { eclodidos } \\
\text { p/postura }\end{array}$ & \\
\hline 12 & & & & & & & \\
\hline $\begin{array}{c}17 / 09 \text { a } 16 / 10 / 81 \\
2^{2}\end{array}$ & 83 & 5,2 & 1.394 & 1.233 & 489 & 5,9 & 39,7 \\
\hline$\frac{16 / 10 \text { a } 15 / 11 / 81}{3^{2}}$ & 117 & 7,3 & 1.559 & 1.387 & 953 & 8,1 & 68,7 \\
\hline $\begin{array}{c}15 / 11 \text { a } 15 / 12 / 81 \\
4^{2}\end{array}$ & 243 & 15,2 & 3.355 & 3.128 & 1.992 & 8,2 & 63,7 \\
\hline $\begin{array}{c}15 / 12 / 81 \text { a } 14 / 01 / 82 \\
5^{2}\end{array}$ & 251 & 15,7 & 3.418 & 3.178 & 2.505 & 10,0 & 78,8 \\
\hline $\begin{array}{c}14 / 01 \text { a } 13 / 02 / 82 \\
6^{2}\end{array}$ & 191 & 11,9 & 2.652 & 2.504 & 1.229 & 6,4 & 49,1 \\
\hline $\begin{array}{c}13 / 02 \text { a } 15 / 03 / 82 \\
7^{2}\end{array}$ & 105 & 6,6 & 2.381 & 2.317 & 1.831 & 17,4 & 79,0 \\
\hline $\begin{array}{c}15 / 03 \text { a } 14 / 04 / 82 \\
8^{2}\end{array}$ & 150 & 9,4 & 3.344 & 3.191 & 1.747 & 11,6 & 54,8 \\
\hline $\begin{array}{c}14 / 04 \text { a } 14 / 05 / 82 \\
9^{2}\end{array}$ & 90 & 5,6 & 2.264 & 2.113 & 649 & 7,2 & 30,7 \\
\hline $\begin{array}{c}14 / 06 \text { a } 13 / 06 / 82 \\
10^{2}\end{array}$ & 90 & 5,6 & 2.063 & 2.058 & 526 & 5,8 & 25,6 \\
\hline $\begin{array}{c}13 / 06 \text { a } 13 / 07 / 82 \\
11^{2}\end{array}$ & 106 & 6,6 & 3.133 & 2.923 & 367 & 3,5 & 12,5 \\
\hline $\begin{array}{c}13 / 07 \text { a } 12 / 08 / 82 \\
12^{9}\end{array}$ & 96 & 6,0 & 3.104 & 2.800 & 387 & 4,0 & 13,8 \\
\hline $13 / 08$ a $11 / 09 / 82$ & 145 & 9,1 & 3.750 & 3.506 & 588 & 4,0 & 16,8 \\
\hline $\bar{X}$ (média) & 138,9 & 8,7 & $2.701,4$ & $2.528,2$ & $1.105,2$ & 7,7 & 44,4 \\
\hline
\end{tabular}


Tabela 3. Fecundidade e fertilidade de $B$. tenagophila $\theta$ B. occidentalis por período de 30 dias, durante os meses de setembro de 1981 a agosto de 1982. Posturas de 16 exemplares

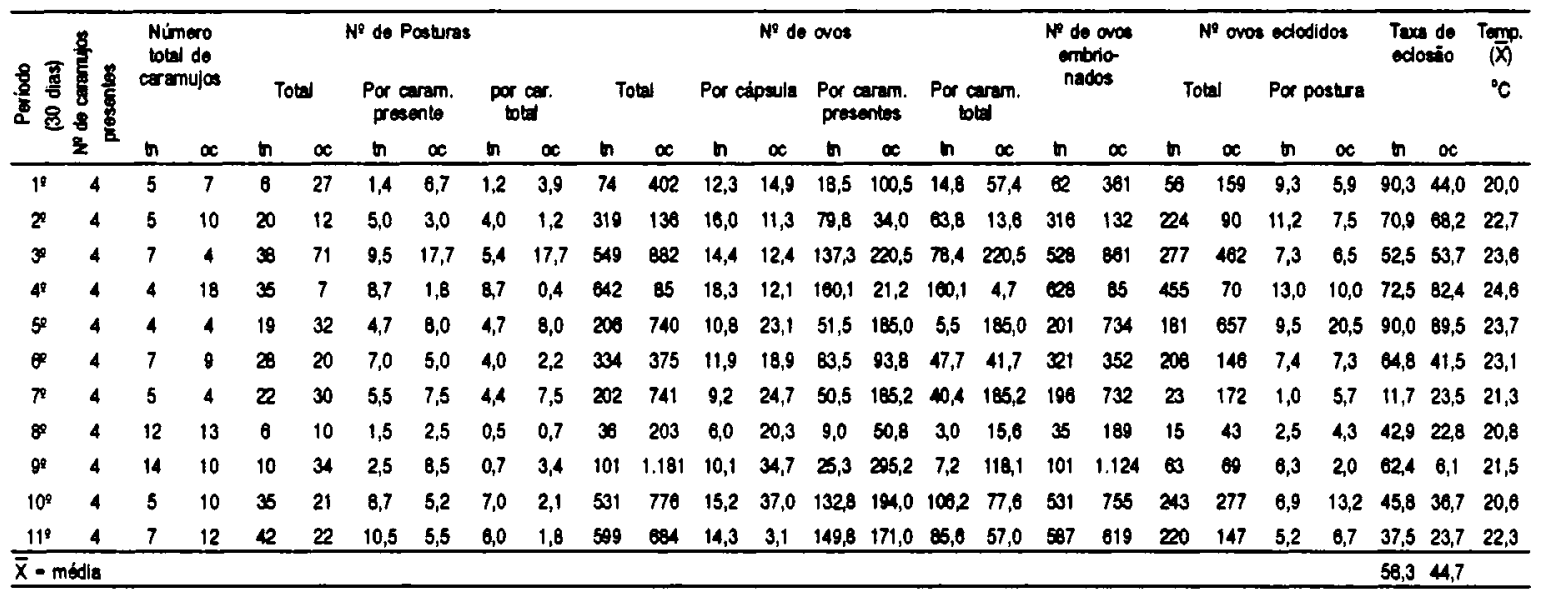

to $=$ B. lonegophila

oc - 8. occidontalis

Se o número total de individuos for levado em consideração alteram-se as informações sobre $B$. occidentalis. O número médio de posturas, por caramujo, em cada período de 30 dias, passa a ser igual a 4,45 , ou seja, 0,15 posturas diárias por caramujo. As últimas cifras não se assemelham, pois, às obtidas inicialmente, quando deixaram de ser levadas em conta as reposições.

Havendo possibilidade de ter a substituição de exemplares influenciado nos resultados, foi necessário analisar o problema com mais cuidado. Para elucidá-lo, foram utilizadas as informações da Tabela 3 e com elas foi procedida a prova de Mann-Whitney, que é um teste comparativo não paramétrico (Siegel $\left.^{11}, 1975\right)$. A primeira hipótese testada buscava verificar se havia diferença significativa quanto ao número de posturas por indivíduo, entre $B$. tenagophila e $B$. occidentalis. $O$ resultado do teste, ao nivel da significância de $5 \%$, mostrou que não existe evidência estatística favorável à suposição feita.

A segunda hipótese testada visava a mostrar a existência de diferença significativa entre o número de ovos por caramujo nas espécies em jogo. $O$ resultado ao nível de significância de $5 \%$ também não levou à evidência estatística em favor dessa diferença. No entanto, o teste aplicado para elucidar uma terceira hipótese, que se refere ao número de ovos por cápsula ovifera em cada espécie, mostrou-se significante ao nível de $5 \%$.

A observação da Tabela 3 permite ainda verificar que durante todo o experimento a mortalidade de $B$. occidentalis foi sempre su- perior à de $B$. tenagophila. No total foram substituidos 101 caramujos da primeira espécie, e 75, da segunda.

No final do estudo, elevou-se o índice de mortalidade dos planorbídeos. Em conseqüência, a taxa de eclosão ilustrada na Figura sofreu certo decréscimo, observável principalmente em $B$. occidentalis, conforme particulariza a Tabela 3 .

Convém ressaltar que muitos dos ovos observados em pleno desenvolvimento estacionavam em uma dada fase, ou mesmo degeneravam, sem que houvesse qualquer alteração evidenciável dos parâmetros ambientais, exceção feita da temperatura.

Houve ainda, se bem que com baixa freqüência, o aparecimento de posturas globosas em ambas as espécies que não aderiram a qualquer substrato e que tornavam muito laboriosa a contagem dos ovos.

\section{Discussão}

\section{Duraçāo do período embrionário}

É difícil comparar os resultados obtidos com os da literatura porque as técnicas de pesquisa variam muito de autor para autor. Apesar disso, os dados aqui referidos para as duas espécies concordam com os observados em Biomphalaria glabrata (Say, 1818), por Luttermoser $^{5}$ (1943) e por Perlowagora Szumlewicz $^{10}$ (1958). Pouco diferem dos obtidos por Sturrock $^{12}$ (1965) em Biomphalaria angulosa Mandahl-Barth, 1958. Sob esse ponto de vista, 


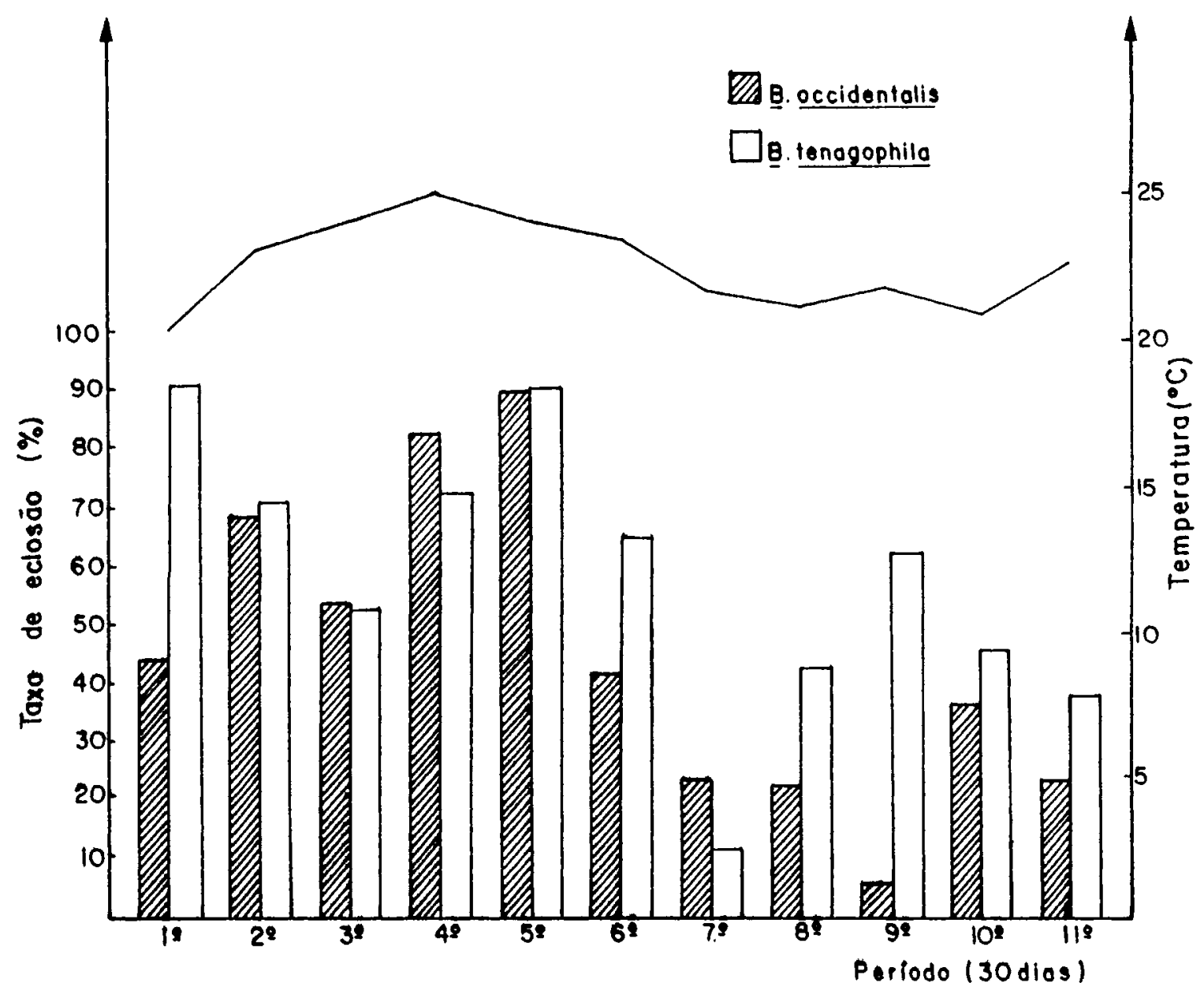

Figura. Comparação da fertilidade de $B$. occidentalis com a $B$. tenagophila em laboratório.

as espécies estudadas em muito se assemelharem às citadas e com elas compartilham da mesma fração de potencialidade para colonizar novos ambientes hídricos.

Convém lembrar que Sturrock e Sturrock $^{13}$ (1972), juntamente com outros autores, admitem ser profunda a influência de pequenas modificaçōes da temperatura sobre a biologia dos planorbídeos. Parece que têm razão porquanto, pelo menos em $B$. occidentalis, pois as máximas e mínimas térmicas mensais coincidiram com os valores extremos do desenvolvimento embrionário.

\section{Fecundidade e Fertilidade}

Foi evidenciada a existência de baixa fecundidade em $B$. occidentalis com relação a $B$. tenagophila, que se traduziu pelo pequeno número de ovos depostos por indivíduo por dia
$(5,62)$, e pelo reduzido número de posturas diárias por caramujo $(0,29)$, dados que conforme já foi dito, procedem da observação de caramujos sem atentar às substituiçōes feitas.

Kawazoe $^{4}$ (1977) observou em B. glabrata os valores respectivos de 13,4 e de 0,65 para os mesmos parâmetros e os de 9,9 e 0,56 , para B. tenagophila.

Houve pouca ou quase nenhuma influência da temperatura sobre a fecundidade, coisa que já fora anteriormente observada por Perlowagora-Szumlewicz ${ }^{10}$, em 1958 quando ao estudar a fecundidade de $B$. glabrata, afirmou: There was no evidence of seasonal rhytm in the reproductivity activity of laboratory reared snails . Em contraposição, Paulini e Camey ${ }^{9}$ (1964) observaram na mesma espécie aumento do número de posturas relacionado com os meses mais quentes do ano.

A fertilidade de $B$. occidentalis mostrou- 
se inferior à de tenagophila. Enquanto na primeira espécie a taxa de eclosão teve o valor de $58,3 \%$ no segundo equivaleu a 44,7 , conforme se observa na Tabela 3.

Há alguma possibilidade de que isso tenha acontecido caso $B$. occidentalis já se encontrasse bem adaptada às condições ambientais de Presidente Prudente, de onde provinha. Enquanto nessa localidade a média das máximas térmicas anuais ascende a $29^{\circ} \mathrm{C}$, em São Paulo equivale a $25^{\circ} \mathrm{C}$. $O$ fato leva a pensar na necessidade de aclimatar previamente $B$. occidentalis antes de introduzi-la com finalidades competitivas em outros biótopos diferentes do original.

A prova estatística foi escolhida por ter havido substituição dos caramujos. Ela é, na verdade, pouco sensível e talvez tenha deixado de evidenciar diversidades sutis entre as espécies consideradas.

De qualquer maneira, o estudo merece ser retomado, modificando algumas das variáveis em jogo, com o objetivo de conhecer a biologia da espécie em maior profundidade.

\section{Conclusões}

Nas condições em que o experimento foi realizado:

- o tempo de desenvolvimento embrionário foi aproximadamente o mesmo em $B$. occidentalis e em B. tenagophila;

- em ambas as espécies, o desenvolvimento embrionário foi mais prolongado nos meses mais frios e, mais curtos nos períodos quentes;

- a fecundidade e a fertilidade de B. occidentalis foram inferiores às de $B$. tenagophila.

Os resultados obtidos sāo válidos para as condiçōes em que o estudo foi realizado e cumpre ter cautela ao buscar generalizá-los.

\section{Agradecimento}

Ao Prof. Moacyr Lobo da Costa Junior, da Faculdade de Saúde Pública de Sāo Paulo, a quem devemos a gentileza da análise estatistica.

ELMOR, M.R.D. et al. [A contribution to the study of the biolgy of Biomphalaria occidentalis Paraense, 1981 and of Biomphalaria tenagophila (d' Orbigny, 1835), under laboratory conditions]. Rev. Saúde públ., S.Paulo, 26: 343-9, 1992.
Specimens of Biomphalaria occidentalis and Biomphalaria tenagophila were reared in S.Paulo, Brazil under laboratory conditions, with a view to know their biological characteristics. Oviposition and eggs/oviposition ratio were recorded over twelve months so as to obtain information on the number of egg-masses per animal and the number of eggs per egg-mass, as also the number of hatched eggs per egg-mass for each period of 30 days, i.e., the hatching-rate per period. The incubation period was about the same for both species, but the oviposition and egg oviposition ratio were greater in $B$. tenagophila.

Keywords: Biomphalaria, physiology. Oviposition.

\section{Referências Bibliográficas}

1. BARNETT, H.C.; BARRY, C; HUFF, R; FARIA, M.S.C.; NOVAES, G.; PESO. M.C.; SANTOS, M.M.L. Reproduction and reproductive control in Biomphalaria snails. In Report International Center for Medical Research, University of Maryland, School of Medicine, 1974 p. 50-8.

2. CHIEFFI, P.P.; MORETTI, I.G.; \& TORNERO, M.T.T. Potencial reprodutivo em condições de laboratório de Biomphalaria glabrata Say (1818) e de Biomphalaria tenagophila d'Otbigny, (1835) originárias do Município de Londrina, Estado do Paraná, Brasil. Rev. Inst. Adolfo Lutz, 37: 65-70, 1977.

3. GENARO, O. Comparação da capacidade ovipositora de Biomphalaria glabrata (Say, 1818), Biomphalaria tenagophila (d'Orbigny, 1835) e Biomphalaria straminea (Dunker, 1848), mantidas em laboratóio. Rev. Soc. bras. Med. trop., 12: 23-7. 1978.

4. KAWAZOE, U. Alguns aspectos da biologia de Biomphalaria glabrata (Say, 1818) e Biomphalaria tenagophila (d'Obigny, 1835) (Pulmonata, Planorbidae). II - Fecundidade e fertilidade. Rev Saúde públ., S.Paulo, 11: 47-64, 1977

5. LUTTERMOSER, G.W. A note on the life cycle of Australorbis glabratus (Say, 1818) Pilsbry, 1934, a snail intermediate host of Schistosoma mansoni. J. Parasitol., 29: 231,1943.

6. OLIVEIRA, M.P.; ALMEIDA, E.L.; VIEIRA. I; OLIVEIRA, M.H.R; Criação de moluscos em terrários e aquários. Lumina spargere, Juiz de Fora, 5: 55-65, 1968.

7. PARAENSE, W.L. Biomphalaria occidentalis sp. n. from South America (Mollusca Basommatophora Pulmonata). Mem. Inst. Oswaldo Cruz, 76: 199-211, 1981.

8. PARAENSE, W.L. \& CORREA, L.R. Unusceptibility of Biomphalaria occidentalis to infection with a strain of Schistosoma mansoni. Mem. Inst. Oswaldo Cruz. 77: 55-8, 1982.

9. PAULINI, E. \& CAMEY, T. Observações sobre a biologia do Australorbis glabratus. II. Influência da temperatura do ambiente sobre a frequência da postura. Rev. bras. Malar. Doenç.trop., 16: 499-504, 1964.

10. PERLOWAGORA-SZUMLEWICZ, A. Studies on the biology of Australorbis glabratus, schistosomabearing Brazilian snail. Rev. bras.Malar. Doenç. trop., 10: 459-529, 1958. 
11. SIEGEL, S. Estatística não paramétrica para as ciencias do comportamento. São Paulo, Mc Graw-Hill do Brasil, 1975.

12. STURROCK, R.F. Studies on the biology of $B$ angulosa Mandahl Barth and on its ability to act as an intermediate host of $S$. mansoni. Ann. trop. Med Parasit. 59: 1-9, 1965.

13. STURROCK, R.F. \& STURROCK B.M. The influence of temperature on the biology of Biomphalaria glabrata (Say), intermediate host os Schistosoma mansoni on St. Lucia, West Indies. Ann trop. Med. Parasit., 66: 385-90, 1972.
14. VAZ, J.F.; ELMOR, M.R.D.; GONÇALVES, LM.C.; ISHIHATA, G.K. Resultados do levantamento planorbidico da área de Presidente Prudente. Estado de São Paulo. Rev. Inst. Med. trop. S.Paulo, 25: $120-6,1983$.

Recebido para publicação em 25.9.1991

Reapresentado em 29.6.1992

Aprovado para publicação em 30.6 .1992 